\title{
EXPLANATION OF CODE
}

\section{SCIENTIFIC SESSIONS}

JS = Joint Sessions (on Sunday, September 5 between the XIIIth International Congress of EEG and Clinical Neurophysiology and the XVth World Congress of Neurology)

$\mathrm{T}=$ Theme Session

$S$ = Symposium

$\mathrm{M}=$ Morning Lecture

\section{POSTER SESSIONS}

\section{PLEASE NOTE EXAMPLE CODING: 1-04-23}

\section{1 = REPRESENTS WHEN THE SESSION TAKES PLACE}

$1=$ Monday Morning Session

2 = Monday Afternoon Session

3 = Tuesday Morning Session

$4=$ Tuesday Afternoon Session

$5=$ Wednesday Morning Session
$6=$ Wednesday Afternoon Session

$7=$ Thursday Morning Session

8 = Thursday Afternoon Session

9 = Friday Moming Session

\section{4 = REPRESENTS THE ABSTRACT'S TOPIC NUMBER FROM TOPIC LIST}

\section{Topic List}

$01=\mathrm{ALS}$ and Related Disorders

02 = Autonomic Nervous System

$03=$ Cerebrovascular Disease

$04=\mathrm{CSF}$

$05=$ Child Neurology

$06=$ Clinical Neurophysiology

$07=$ Cognitive and Behavioural Neurology

$08=$ Critical Care/Emergency Neurology

$09=$ Degenerative Diseases

$10=$ Dementia and Aging

11 = Epilepsy

$12=$ Ethics

13 = Genetic Diseases

14 = Headache and Pain

$15=$ History of Neurology

$16=$ Infectious Diseases
$17=$ Movement Disorders

$18=$ MS and Related Disorders

$19=$ Muscle Diseases

$20=$ Myasthenia and Related Diseases

21 = Neuroepidemiology

22 = Neuro-imaging

23 = Neuro-immunology

$24=$ Neurological Education

$25=$ Neuro-oncology

26 = Neuro-ophthalmology

27 = Neuropharmacology

$28=$ Peripheral Nerve Diseases

$29=$ Rehabilitation

$30=$ Trauma

$31=$ Other

23 = REPRESENTS THE ABSTRACT NUMBER FOR SPECIFIC SESSION AND TOPIC

In this example: 1-04-23 indicates $A$ bstract 23 presented at the Monday morning Poster Session on CSF. 